TAed.

\author{
FORSCHUNG - AUSBILDUNG - WEITERBILDUNG \\ Bericht Nr. 45
}

QUALITY CONTROL OF ARTIFICIAL FABRICS

\author{
Peter Hackh \\ Fachbereich Mathematik \\ Universität Kaiserslautern \\ Postfach 3049 \\ D - 6750 Kaiserslautern
}

\begin{abstract}
UNIVERSITÄT KAISERSLAUTERN
Fachbereich Mathematik

Erwin-Schrödinger-Straße

D - 6750 Kaiserlautern
\end{abstract}

Juni 1990 


\title{
QUALITY CONTROL OF ARTIFICIAL FABRICS
}

\author{
Peter Hackh
}

Abstract: Some new approaches to measure irregularities of artificial tissues are introduced.

\section{Introduction}

One problem which arises in different branches of industry is to find objective measures for the irregularities resp. defects of tissues like clouds, stripes etc. (see [2] and [3]). Often the valuation of the fabric is done in the following way:

It is put in front of a black background and several persons judge upon their personal visual impression on it, that means: they tell how even it appears to them.

Therefore: In order to find an objective procedure which is meant to substitute the above described subjective one, one is in this case not allowed to resort to measurements of the thickness of the fabric, but for instance to the transmission rates of a laser beam; however this is not the crucial point. One may assume that there are some tracks along the tissue, or there is some raster picture, - with for instance $512 \times 512$ pixels. The idea is that the distribution which is given by such a record has to be compared with a "uniform distribution"; we wish to find a number which measures the deviation from the uniform distribution "given by" the mean transition rate. In this way we hope to find a measure for the "cloudiness" of the fabric ([3]).

As long as it concerns the problem of stripes resp. anisotropy, we have made a 2d-Wavelet-Analysis for some tissues, which allows to detect edges or to find directions for the fibres which are preferred in the fabric, but in this paper we wish to discuss only the first point. 
If one looks at a certain tissue the idea is not too far away from thinking of it being a cluster, a pattern or even a mathematical measure: The measure of a certain subset is given by the rate of light, which passes through this area. In order to be able to compare different fabrics which may have different thickness, we have to normalize our measurements:

The measure of a certain area is now given by the ratio of the light which passes through this part and the light which passes through the whole tissue. Here one is not obliged to think of the raster picture but of the "one dimensional record" too: The light which passes through the whole fabric is in this case again the sum of all intensity values.

But what is now about a natural concept for the distance of (such) measures, for we like to compare our measures/patterns with the uniform distribution (Figure 1 and 2)?

I wish to present only two distance concepts, the Bounded Lipschitz Distance (BLD) and the Interval Discrepancy.

\section{$2.1 \quad$ BLD:}

If one has a metric space $(X, d)$ (together with its $\sigma$-Algebra of Borel sets on it) and two probability measures $\mu$ and $\nu$ on $\mathrm{X}$, then the BLD of $\mu$ and $\nu, \rho(\mu, \nu)$, can be defined in the following way:

$$
\rho(\mu, \nu):=\sup \left\{\left|\int \mathrm{fd}(\mu-\nu)\right|:|\mathrm{f}(\mathrm{x})-\mathrm{f}(\mathrm{y})| \leq \mathrm{d}(\mathrm{x}, \mathrm{y}), \begin{array}{l}
\mathrm{f}: \mathrm{X} \rightarrow \mathbb{R} \\
\text { bounded }
\end{array}\right\}
$$

If we reduce this now to the 1 -dimensional discrete case, we get

$$
\sup \left\{\left|\sum f_{i} \cdot m_{i}\right|:\left|f_{i}-f_{i+1}\right| \leq 1\right\} \text {, }
$$

- the $\mathrm{m}_{\mathrm{i}}$ are now the normalized measurements; this means: Each original value $M_{i}$ is divided by $\sum M_{i}$ and at least $1 / n-n$ the number of values - is subtracted.

That is: The two measures we wish to compare are already involved. (It is easy to see that we can forget about the boundedness condition for $f$ in equation (1)!)

This problem is not so complicate, - it is a problem of linear programming, but if one regards about 5000 measurements for one 
calculation - and we have to do so - the simplex algorithm lasts much too long. Rote ([4]) found a relativ fast algorithm for a more general class of BLD's in the discrete case, but it is still too slow to handle these high dimensional cases which we would like to look at. It is quite astonishing that there exists nevertheless a very simple formula resp. algorithm for our special BLD: We have

$$
\sup \left\{\left|\sum_{i=1}^{n} f_{i} \cdot m_{i}\right|:\left|f_{i}-f_{i+1}\right| \leq 1\right\}=\sum_{i=1}^{n}\left|\sum_{j=1}^{i} m_{j}\right| \text {. }
$$

Wick proved a proposition for which this equation is only the discrete special case (see [5]), - to appear in "Archiv der Mathematik" :

Given a Uniformity Class

$\mathrm{U}:=\{\Phi \in \mathrm{C}[0,1]: 0 \leqslant \Phi \leqslant 1,|\Phi(\mathrm{x})-\Phi(\mathrm{y})| \leqslant|\mathrm{x}-\mathrm{y}| \forall \mathrm{x}, \mathrm{y} \in \mathbb{R}\}$

$\mu$ and $\nu$ probability measures on $[0,1]$, then

$$
\rho(\mu, \nu)=\mathrm{D}^{1}(\mu, \nu),
$$

where

$$
\rho(\mu, \nu):=\sup _{\Phi \in U}|(\mu-\nu)(\Phi)| \text { and } D^{1}(\mu, \nu):=\int_{0}^{1}|(\mu-\nu)([0, \mathrm{x}])| \mathrm{dx}
$$

Proof: Only for (3), the discrete case,

1. $\quad \sum_{i=1}^{n} f_{i} \cdot m_{i}=\sum_{i=1}^{n}\left\{\sum_{j=1}^{i} m_{j}\right\}\left(f_{i}-f_{i+1}\right), \quad f_{n+1}:=0$;

therefore

$$
\left|\sum_{i=1}^{n} f_{i} \cdot m_{i}\right| \leq \sum_{i=1}^{n}\left|\sum_{j=1}^{i} m_{j}\right|
$$

2. $\sum_{i=1}^{n}\left|\sum_{j=1}^{i} m_{j}\right|$ again is an expression of the form $\sum g_{i} \cdot m_{i}$ with $\quad g_{i}-g_{i+1} \mid \leq 1$, because $\left|\sum_{j=1}^{i} m_{j}\right|=\alpha_{i} \cdot \sum_{j=1}^{i} m_{j}$ with 
$\alpha_{i}=\operatorname{sign}\left(\sum_{j=1}^{i} m_{j}\right)$

This implies

$\sum_{i=1}^{n}\left|\sum_{j=1}^{i} m_{j}\right|=\sum_{i=1}^{n} \alpha_{i} \sum_{j=1}^{i} m_{j}=\sum_{j=1}^{n}\left(\sum_{i \geq j} \alpha_{i}\right) m_{j}$

and with $g_{j}:=\sum_{i \geq j} \alpha_{i}$ one gets $\left|g_{j}-g_{j+1}\right|=\left|\alpha_{j}\right| \leq 1$.

Therefore the right hand side of (3) is the maximum of the set which we are interested in. It is clear that

(4). $\sup \left\{\left|\sum_{i=1}^{n} f_{i} \cdot m_{i}\right|:\left|f_{i}-f_{i+1}\right| \leq 1 / n\right\}=\frac{1}{n} \cdot \sum_{i=1}^{n}\left|\sum_{j=1}^{i} m_{j}\right|$.

This equation now yields two ideas:

$\alpha)$ We have a more precise idea of what our special BLD really is

- it is the mean value of the absolute values of all partial sums of our normalized measurements, - therefore it is perhaps not an appropriate tool for our analysis.

B) Nevertheless, if one really wishes to use it, you have a very quick algorithm at your disposal, which should be the optimal one too:

$\operatorname{lip}:=$ help $:=0$;

for $i=1, \ldots, n$ :

begin

help $=$ help + value $[i] ; / *$ "value" is array of measure-

if (help $>0)$

$\left.\begin{array}{l}\text { lip }=\text { lip }+ \text { help; } \\ \operatorname{lip}=\operatorname{lip}-\operatorname{help} ;\end{array}\right\} \quad$ lip $=$ lip $+\operatorname{sign}($ help $) *$ help

end;

lip $=1 \mathrm{ip} / \mathrm{n} ; / *$ the above mentioned second version of the discrete BLD $\longrightarrow$ formula (4) */

\subsection{Discrepancy}

$\mathrm{D}^{1}$ mentioned above is the so called $\mathrm{L}^{1}$-Discrepancy; there is a great class of other discrepancy concepts, - we only wish 
to mention the Extreme Discrepancy $\sup _{x} I(\mu-\nu)([0, x]) I$ and the Interval Discrepancy $\sup _{0 \leq \mathrm{x}, \mathrm{y}} \mid(\mu-\nu)([\mathrm{x}, \mathrm{y}]) 1$, - here only written down for the continuous $1 \mathrm{~d}$-case, $\mathrm{X}=[0,1], \mu$ and $\nu$ our probability measures as above (see [1]).

We have applied the discrete version of the Interval Discrepancy to the $1 \mathrm{~d}$ - and $2 \mathrm{~d}$-case of pictures, for it seems to be a very natural concept: Applying it means: to look for biggest "holes" or "mountains" (connected areas with great deviations from the mean value of the intensity), - and many little holes are better than only a few big holes; see figure 1 and 2 .

2.2.1 Calculations of the Interval Discrepancy in the 1d-case. If $\left(m_{i}\right)_{1 \leqslant i \leqslant n}$ is our "normalized" record, we are interested in

$$
\max _{1 \leqslant i \leqslant j \leqslant n}\left|F_{i}-F_{j}\right| \text { with } F_{i}:=\sum_{\nu=1}^{i} m_{\nu} .
$$

We can write this in a more symmetric way: $\max _{1 \leq i, j \leqslant n} \mid F_{i}-F_{j} l$, - and this is the simple difference $\max \left(\mathrm{F}_{i}\right)-\min \left(\mathrm{F}_{i}\right)$; this equation involves a very trivial fast algorithm:

One has to calculate the partial sums and to update maximum and minimum in each step. This is a "linear" algorithm and in this sense it is optimal; one has to "scan" each value for only one time. The idea to do it in this way goes back to Rote ([4]) too.

\section{2 .2 Interval Discrepancy in the 2d-case}

In this case it seems to be impossible to find a similar nice trick as in the $1 \mathrm{~d}$-case, - the situation is quite different. One possibility would be: Reduce it in a certain way to the 1d-case:

a) Substitute each row of our, say $512 \times 512$-matrix by the sequence of its partial sums.

b) Calculate now sums of increasing numbers of neighboured rows.

c) Determine the discrepancies of these vectors.

d) The maximum of these discrepancies is the discrepancy of the "picture". 
We have chosen another way: We determine the subarrays with the maximum $(\max )$ resp. minimum (min) sum of its elements; Max $\{\max ,-m i n\}$ is the discrepancy which has to be determined. In what follows, we again assume, that the elements of our matrix are just the normalized measurement values (this means: the equidistribution is already involved in these numbers).

Some remarks now w.r.t. the crucial part of the algorithm:

Row by row (original picture matrix "pic"), for each "part" pic[k][j],...,pic[k][j+i] - k-th row! we determine the sum of the elements of that subarray among all subarrays with this "part" as last row, which has the greatest/smallest sum.

The algorithm works as follows:

We use the two upper triangular matrices $D=\left(d_{i j}\right), \underline{D}=\left(\underline{d}_{i j}\right)$, which have to be updated in each step (maxima are stored in $D$, minima in $\underline{D})$ :

After step $k$ (which "belongs to" the k-th row of the pic-matrix) element $d_{i j}$ resp. $\underline{d}_{i j}$ is the maximum resp. minimum of the set of the sums of all subarrays with the last row constituted by the elements pic[k][j] until pic[k][j+i]; we allow height=0 for our rectangulars.

Initialization: $\mathrm{D}^{(0)}=\underline{\mathrm{D}}^{(\mathrm{O})}=0$; $\max =\operatorname{mi} \cdot \mathbf{n}=0$;

Recursion: for $(\mathrm{k}=1, \ldots, \mathrm{n})$

begin

$$
\begin{aligned}
& d_{i j}^{(k)}=\max \left(0, d_{i j}^{(k-1)}+\sum_{\nu=1}^{i} \operatorname{pic}[k][\nu+j]\right) ; \\
& \underline{d}_{i j}^{(k)}=\min \left(0, \underline{d}_{i j}^{(k-1)}+\sum_{\nu=1}^{i} \operatorname{pic}[k][\nu+j]\right) ; \\
& \text { if }\left(d_{i j}^{(k)}>\max \right) \max =d_{i j}^{(k)} ; \\
& \quad \text { if }\left(\underline{d}_{i j}^{(k)}>\min \right) \min =\underline{d}_{i j}^{(k)} ; \\
& \text { end; } \\
& \text { discrepancy }:=\operatorname{MAX}(\max ,-\min ) ;
\end{aligned}
$$

Question: What is the optimal algorithm? 
3. 1 1d-case:

In some cases it is possible for instance to identify the cleaning periods for the air channels, but in the case of other tissues it seems to be quite impossible to detect such phases by means of discrepancy analysis: Maybe that the increasing pollution of the air channel appears here only as an increasing thickness of the fabric; perhaps discrepancy is not a sensitive enough "tool" for our task and we have to look for other mathematical models. Nevertheless: Discrepancy measures some feature of the fabric, although it does not always coincide with what the company calls "cloudiness". Here work is still on progress.

\section{$3.22 \mathrm{~d}-$ case :}

We have calculated the 2d-interval-discrepancy for different tissues; the results coincide fairly well with the visual impression (great values - bad quality, less even / small values - good quality, more even), apart from lacking in some "selectivity": There are tissues which look very similar, and the difference of the values is of an order not very different from that of the discrepancies of two fabrics which look rather more unequal.

Maybe that the procedure is still too coarse and one may be well advised by applying an "isotropic" discrepancy, an alternative concept in which intervals have to be substituted by "convex sets" or a more general discrepancy concept which deals with all "connected sets". But this is a question of time too: Such calculations would be much more elaborate and would last very long; this is again a problem of finding a fast algorithm.

\section{References}

[1] Kuipers, L., Niederreiter, H.: Uniform Distribution of Sequences, 1974, Wiley, New York

[2] Neunzert, H., Wetton, B.: Pattern Recognition Using Measure Space Metrics, preprint no. 28, Arbeitsgruppe Technomathematik, Universität Kaiserslautern, 1987

[3] Neunzert, H.: Industrial Mathematics: General Remarks and Some Case Studies, preprint no. 34, Arbeitsgruppe Technomathematik, Universität Kaiserslautern, 1989 
[4] Rote, G.: personal communication 1988

[5] Wick, J.: personal communication 1989

Peter Hackh

Department of Mathematics

University of Kaiserslautern

Erwin-Schrödinger-Straße

D - 6750 Kaiserslautern 


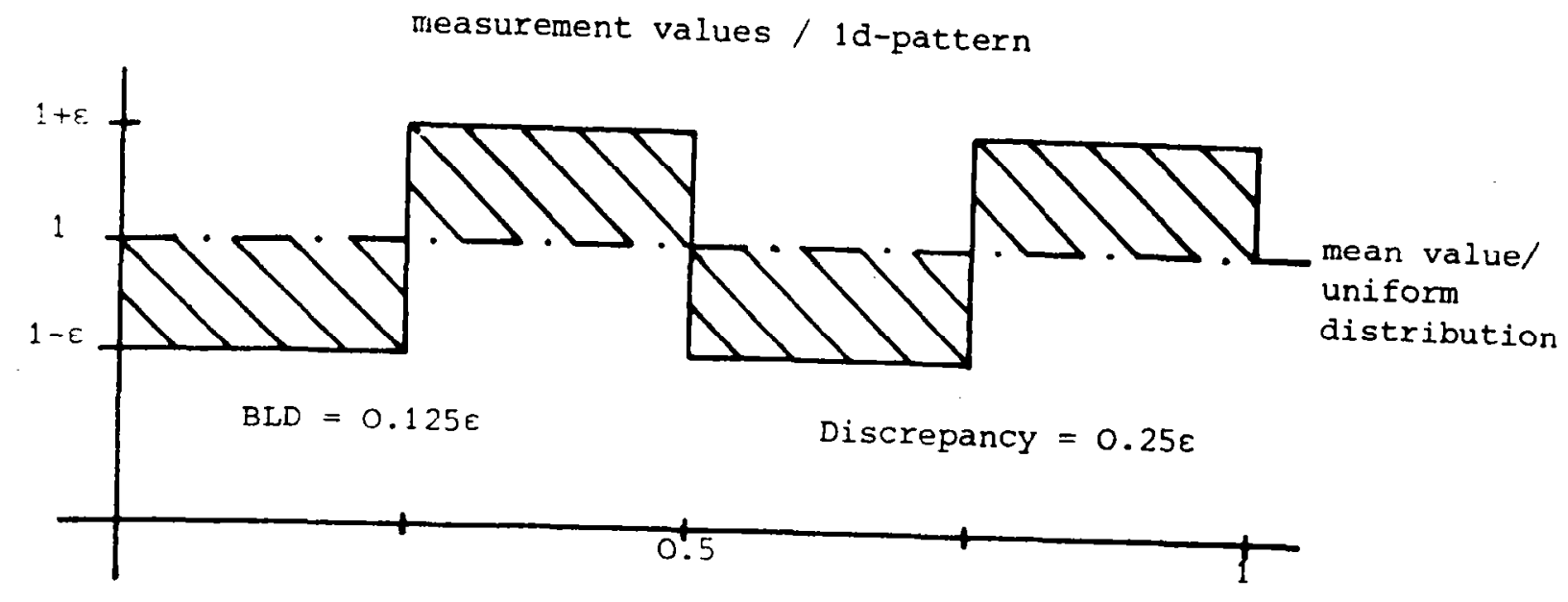

Figure 1

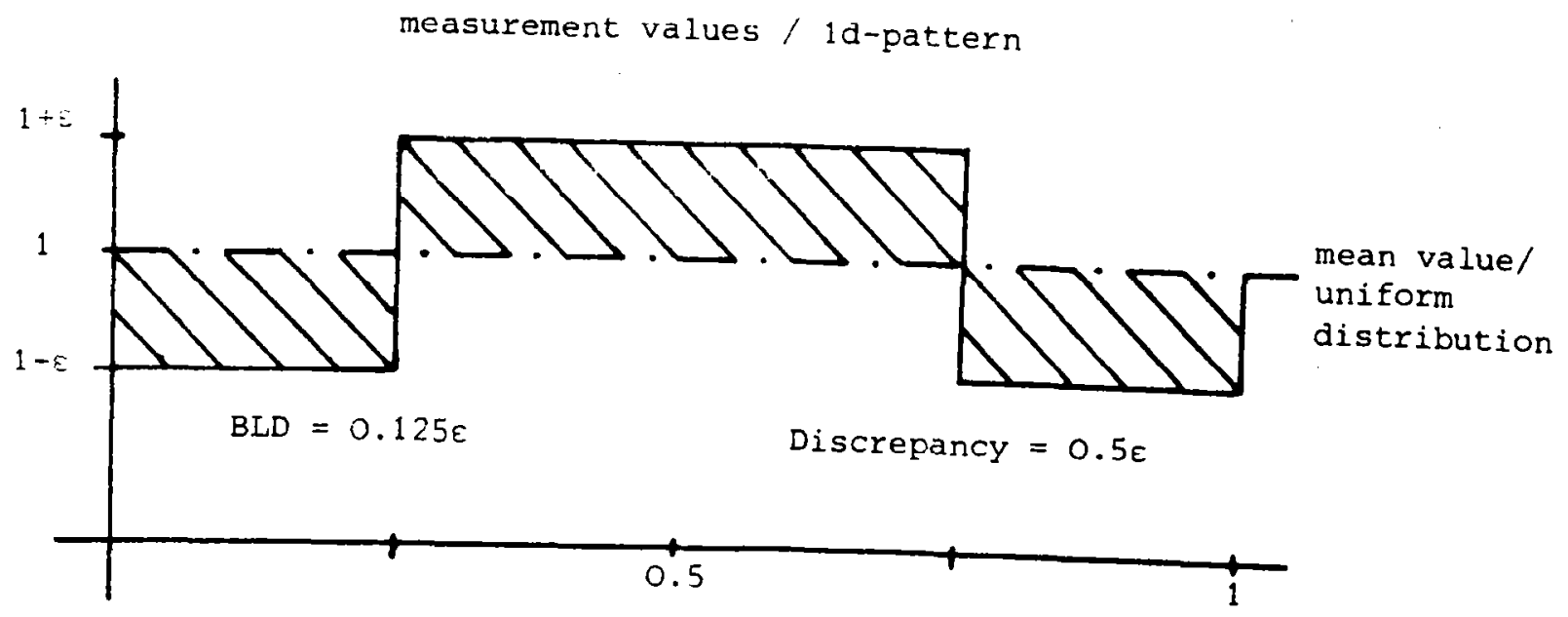

Figure 2 